\title{
Dispositivo de segurança para assistência ventilatória: Conectony - minimizando riscos no contexto COVID-19
}

\author{
Safety device for ventilatory assistance: Conectony - minimizing risks in the COVID-19 context \\ Dispositivo de seguridad para la asistencia ventiladora: Conectonía: minimización de riesgos en el contexto de COVID-19
}

\begin{abstract}
RESUMO
Objetivo: Apresentar o relato de experiência da ação colaborativa que possibilitou, a partir da ideia de inovação proposta pelo autor, a confecção do CONECTONY. Este trata-se de um dispositivo de segurança composto por dois conectores interligados por um segmento de tubo flexível que tem por objetivo prolongar a cânula de traqueostomia e possibilitar o clampeamento com uma pinça forte, permitindo atender as recomendações das principais sociedades de assistência à saúde, nacionais e internacionais, no que diz respeito a manipulação da via aérea avançada nos pacientes acometidos por COVID-19. Metodologia: Estudo descritivo tipo relato de experiência fundamentado no processo de Design thinking. Relato de Experiência: A partir das expecificações técnicas e medidas fornecidas pelo autor, um professor de Engenharia Metalúrgica e de Materiais realizou a constução do desenho técnico das peças para impressão em formato 3D. 0 protótipo impresso em 3D demonstrou perfeita adaptabilidade à cânula de traqueostomia e aos conectores e circuitos de assistência ventilatória. Considerações finais: Incorporar a utilização do dispositivo CONECTONYà prática assistencial e ao cuidado de Enfermagem aos pacientes traqueostomizados, tem potencial para conferir maior segurança aos profissionais de saúde, minimizando risco de contaminação por COVID-19.

DESCRITORES: Infecções por Coronavirus; Respiração Artificial; Difusão de Inovações; Cuidados Críticos; Cuidados de Enfermagem.
\end{abstract}

\section{ABSTRACT}

Objective: To present the experience report of the collaborative action that made possible, based on the idea of innovation proposed by the author, the making of CONECTONY. This is a safety device consisting of two connectors interconnected by a segment of flexible tube that aims to extend the tracheostomy cannula and enable clamping with a strong clamp, allowing to meet the recommendations of the main healthcare companies, national and international, regarding the manipulation of the advanced airway in patients affected by COVID-19. Methodology: Descriptive study like an experience report based on the Design thinking process. Experience Report: Based on the technical specifications and measures provided by the author, a professor of Metallurgical and Materials Engineering carried out the construction of the technical design of the parts for printing in 3D format. The 3D printed prototype demonstrated perfect adaptability to the tracheostomy cannula and to the ventilatory assistance connectors and circuits. Final considerations: Incorporating the use of the CONECTONY device into healthcare practice and nursing care for tracheostomized patients, has the potential to provide greater safety to health professionals, minimizing the risk of contamination by COVID-19 DESCRIPTORS: Coronavirus Infections; Respiration Artificial; Diffusion of Innovation; Critical Care; Nursing Care.

\section{RESUMEN}

Objetivo: Presentar el informe de experiencia de la acción colaborativa que hizo posible, en base a la idea de innovación propuesta por el autor, la realización de CONECTONY. Este es un dispositivo de seguridad compuesto por dos conectores interconectados por un segmento de tubo flexible que tiene como objetivo extender la cánula de traqueotomía y permitir la sujeción con una abrazadera fuerte, lo que permite cumplir con las recomendaciones de las principales compañías de atención médica, nacional e internacional, sobre la manipulación de la vía aérea avanzada en pacientes afectados por COVID-19. Metodología: estudio descriptivo como un informe de experiencia basado en el proceso de pensamiento de diseño. Informe de experiencia: Basado en las especificaciones y medidas técnicas proporcionadas por el autor, un profesor de Ingeniería Metalúrgica y de Materiales llevó a cabo la construcción del dibujo técnico de las piezas para imprimir en formato 3D. El prototipo impreso en 3D demostró una adaptabilidad perfecta a la cánula de traqueotomía y a los conectores y circuitos de asistencia ventilatoria. Consideraciones finales: la incorporación del uso del dispositivo CONECTONY en la práctica de atención médica y la atención de enfermería para pacientes traqueostomizados, tiene el potencial de proporcionar mayor seguridad a los profesionales de la salud, minimizando el riesgo de contaminación por COVID-19 DESCRIPTORES: Infecciones por Coronavirus; Respiración Artificial; Difusión de Innovaciones, Cuidados Críticos; Atención de Enfermería.

RECEBIDO EM: 02/05/2020 APROVADO EM: 05/05/2020 


\section{Tony de Oliveira Figueiredo}

Mestre em Enfermagem. MBA Executivo. Especialista em Administração Hospitalar, Cirurgia Cardiovascular e Circulação Extracorpórea. Diretor da Divisão de Enfermagem do HUCFF/UFRJ. Enfermeiro Assistencial da Unidade Cardiointensiva do HUAP/ UFF. RJ/Brasil. https://orcid.org/0000-0003-2259-1510

\section{Celio Albano da Costa Neto}

Mestre em Engenharia Metalúrgica e de Materiais. Doutor em Engenharia Metalúrgica e de Materiais pelo Illinois Institute of Technology, Chicago, Illinois, USA. Professor Associado do Departamento/Programa de Engenharia Metalúrgica e de Materiais. Universidade Federal do Rio de Janeiro (POLI/COPPE/UFRJ). RJ/Brasil. https://orcid.org/0000-0003-3158-1795

\section{INTRODUÇÃO}

A ntes da pandemia de COVID-19, cinco coronavírus já eram conhecidos por infectar seres humanos, entre eles o SARS- CoV, responsável pelo surto global em 2003. Os coronavírus são particularmente patogênicos em seres humanos e estão associados a difícil manejo, alta mortalidade e sérias implicações para a saúde pública ${ }^{(1)}$.

Urge a necessidade de produção estudos para fundamentar, com evidências científicas, tanto o gerenciamento, manejo clínico e cuidados, quanto a produção de inovações e soluções tecnologias que possam ser rapidamente utilizadas no enfrentamento da COVID-19.

No contexto da pandemia por coronavírus, em pacientes que necessitam de uma via aérea avançada para assistência ventilatória, preconiza-se que, no momento da intubação orotraqueal (IOT), a inserção do tubo orotraqueal (TOT) se dê com o mesmo clampeado por uma pinça forte. A orientação é que esta pinça deva ser utilizada em todos os momentos que se faça necessária a desconexão do TOT do ventilador mecânico. O objetivo deste clampeamento é evitar a aerossolização de vírus no ambiente e, por consequência, evitar a contaminação dos profissionais de saúde ${ }^{(2-4)}$.

Nos casos clínicos que evoluem com dificuldade de retirada do paciente de prótese ventilatória, quando ocorre a necessidade de prolongar o tempo de ventilação mecânica, pode ser necessária a confecção de uma traqueostomia. Os modelos convencionais de cânu-
Antes da pandemia

de COVID-19,

cinco coronavírus

já eram conhecidos

por infectar seres

humanos, entre

eles o SARS-CoV,

responsável pelo

surto global em

2003. Os coronavírus

são particularmente

patogênicos em

seres humanos $\mathrm{e}$

estão associados a

difícil manejo, alta

mortalidade e sérias

implicações para a

saúde pública ${ }^{(1)}$. las de traqueostomia não permitem o clampeamento preconizado, predispondo profissionais de saúde a riscos tanto no momento da confecção cirúrgica, quanto nos casos de desconexão do circuito.

Os avanços tecnológicos são uma realidade no mundo atual, atuar utilizando ferramentas tecnológicas que propiciem a facilitação do trabalho e a otimização de tempo e recursos, incorporando diferentes modalidades de interatividade por meio do uso de tecnologias, são competências necessárias e imprescindíveis de serem aprendidas e continuamente aplicadas nos processos de trabalho ${ }^{(5)}$.

O desenvolvimento de projetos colaborativos, interativos e interdisciplinares pode ser facilitado por meio de utilização de Tecnologias de Comunicação e Informação (TIC). A interatividade garante agilidade às respostas necessárias, permitindo estabelecer mecanismos de comunicação rápida, eficiente e segura. Uma das possibilidades refere-se ainda à gestão remota de projetos, propiciando o planejamento, desenvolvimento, monitoramento e avaliação $^{(6)}$.

Neste sentido, este estudo tem por objetivo apresentar o relato de experiência da ação colaborativa que possibilitou, a partir da ideia de inovação proposta pelo autor, a confecção do CONECTONY. Este trata-se de um dispositivo de segurança composto por dois conectores interligados por um segmento de tubo flexível que tem por objetivo prolongar a cânula de traqueostomia e possibilitar o clampeamento com uma pinça forte, permitindo atender as recomendações das princi- 
pais sociedades de assistência à saúde, nacionais e internacionais, no que diz respeito à manipulação da via aérea avançada nos pacientes acometidos por COVID-19.

Destacamos a relevância deste estudo tanto na descrição do processo colaborativo de construção do conhecimento, quanto na descrição da construção tecnológica do dispositivo em si, que confere segurança ao cuidado minimizando riscos aos profissionais de saúde.

\section{METODOLOGIA}

Estudo descritivo, tipo relato de experiência de inovação tecnológica, no qual foi abordado o processo colaborativo de construção de um dispositivo de segurança para assistência à saúde a partir de processos e fundamentos do Disign Thinking.

O Design Thinking é um método prático-criativo de solução de problemas, que tem potencial para transformar a produção de conhecimentos e soluções. Desenvolve um processo metodológico intencional para se chegar a soluções criativas, práticas e criar impactos positivos. $\mathrm{O}$ processo de modo geral envolve cinco etapas: empatizar, definir, idear, prototipar e testar. Por meio destas, os problemas vão sendo elucidados por meio do diálogo e da observação e as ideias vão tomando forma até se concretizarem em uma solução, que será colocada em prática ${ }^{(7)}$.

A construção tecnológica do dispositivo CONECTONY resultou do relatório de ideação e de documentos da pesquisa definidos a partir da experiência profissional do autor, Enfermeiro com mais de 25 anos de atividade em Unidades de Terapia Intensiva e Alta Complexidade, com produção técnica e científica na área.

A empatia com a situação de risco dos profissionais de saúde no momento do cuidado a pacientes acometidos pela pandemia de COVID-19 leva à constante reflexão sobre a revisão de processos assistenciais e utilização de tecnologias e foi determinante na ideia de melhoria.

\section{Figura 1. Plotagem do dispositivo CONECTONY. Rio de Janeiro, RJ, Brasil, 2020.}

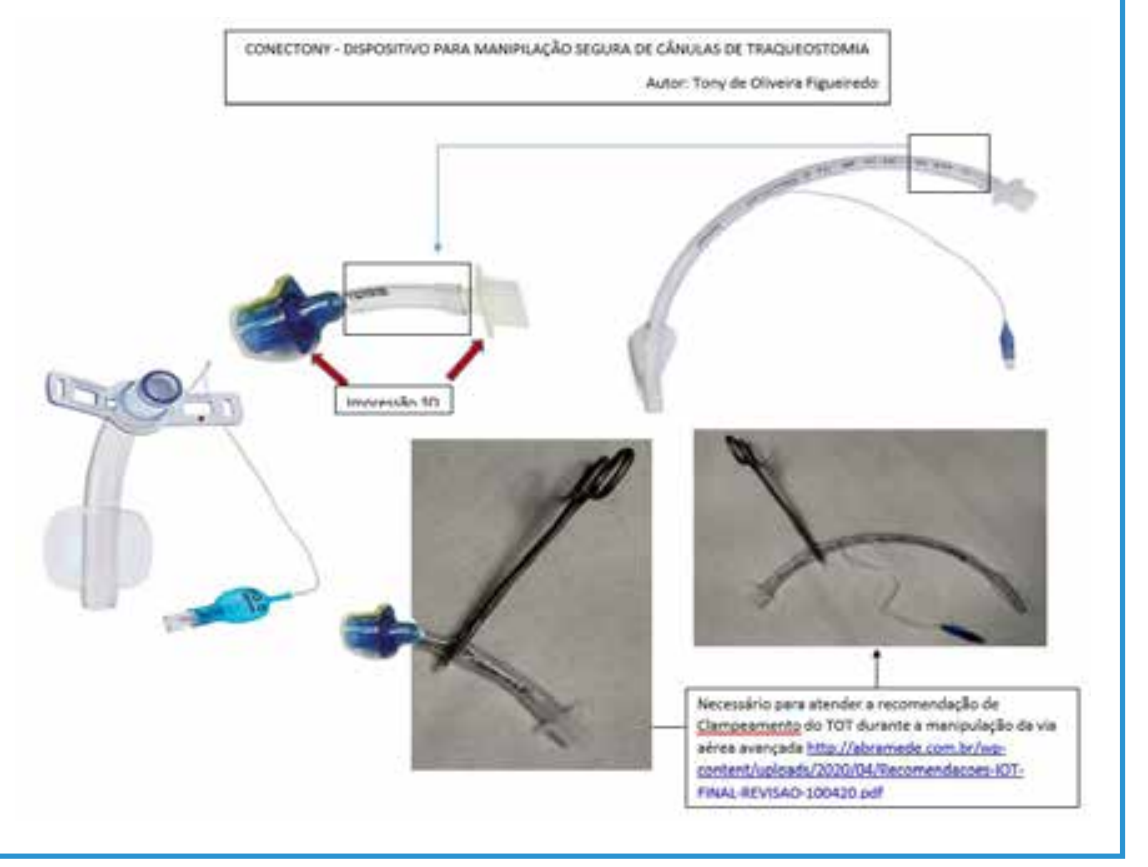

A plotagem do projeto por meio de imagens permitiu à área técnica o perfeito entendimento da ideia, bem como de sua relevância. A apresentação foi construída com base em imagens disponíveis na Internet e recursos do programa Microsoft Word $^{\circledR}$ (Figura 1). O autor acompanhou e prestou consultoria em todas as etapas de desenvolvimento e ficou responsável pelos testes de funcionalidade do dispositivo.

O relatório de ideação e a justificativa para implementação do conceito, foram apresentados à àrea ténica por meio de vídeo (disponível em: https:// youtu.be/8EY_ahhjpIE) enviados por meio de mensagens no aplicativo para Internet/Whatsapp e discutidos no dia 21 de abril de 2020, no Grupo de Trabalho (GT) Multidisciplinar para Enfrentamento da COVID-19 da Universidade Federal do Rio de Janeiro (UFRJ).

$\mathrm{O}$ autor expôs sua intenção, em nome do bem comum e da vida humana, considerando a urgência na produção de soluções no enfrentamento e prevenção da COVID-19, de permitir a disseminação do conceito, bem como disponibilizar publicamente as especificações para impressão do dispositivo em formato 3D. Houve a orientação por integrantes do GT quanto ao registro de propriedade intelectual por parte do autor, permitindo a reprodução da tecnologia, desde que referenciado o autor/fonte de desenvolvimento. No mesmo dia, 21 de abril de 2020, foi realizado o registro de propriedade intelectual no site https://creativecommons.org/.

\section{RELATO DE EXPERIÊNCIA}

A partir das expecificações técnicas e medidas fornecidas pelo autor, um professor de Engenharia Metalúrgica e de Materiais do Instituto Alberto Luiz Coimbra de Pós-Graduação e Pesquisa de Engenharia, da Universidade Federal do Rio de Janeiro (COPPE/UFRJ) realizou a constução do desenho técnico das peças para 


\section{artigo}

\section{Figura 2. Conector 1. Rio de Janeiro, RJ, Brasil, 2020}

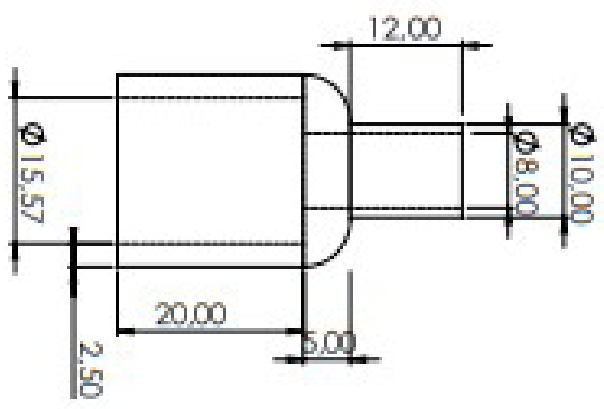

Figura 3. Conector 2. Rio de Janeiro, RJ, Brasil, 2020

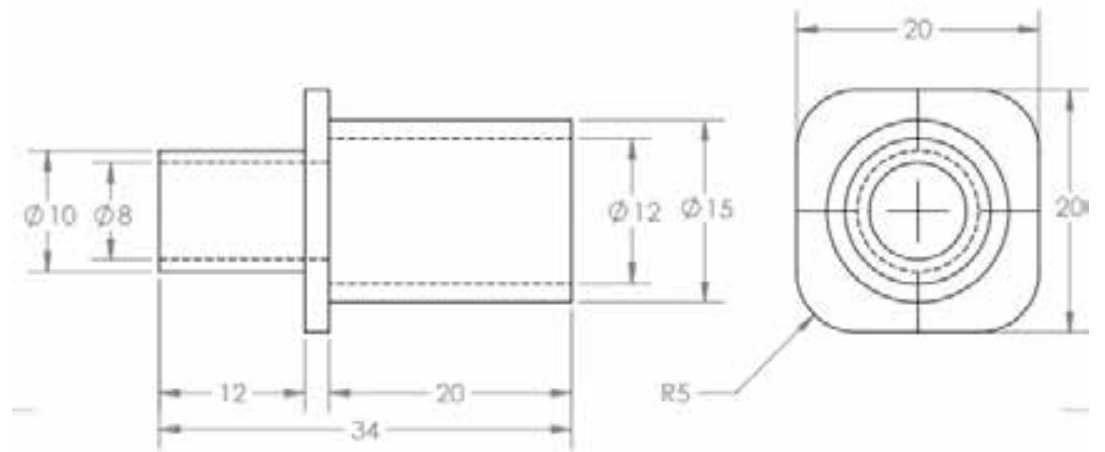

Figura 4. Conectores 1 e 2 impressos em 3D. Rio de Janeiro, RJ, Brasil, 2020

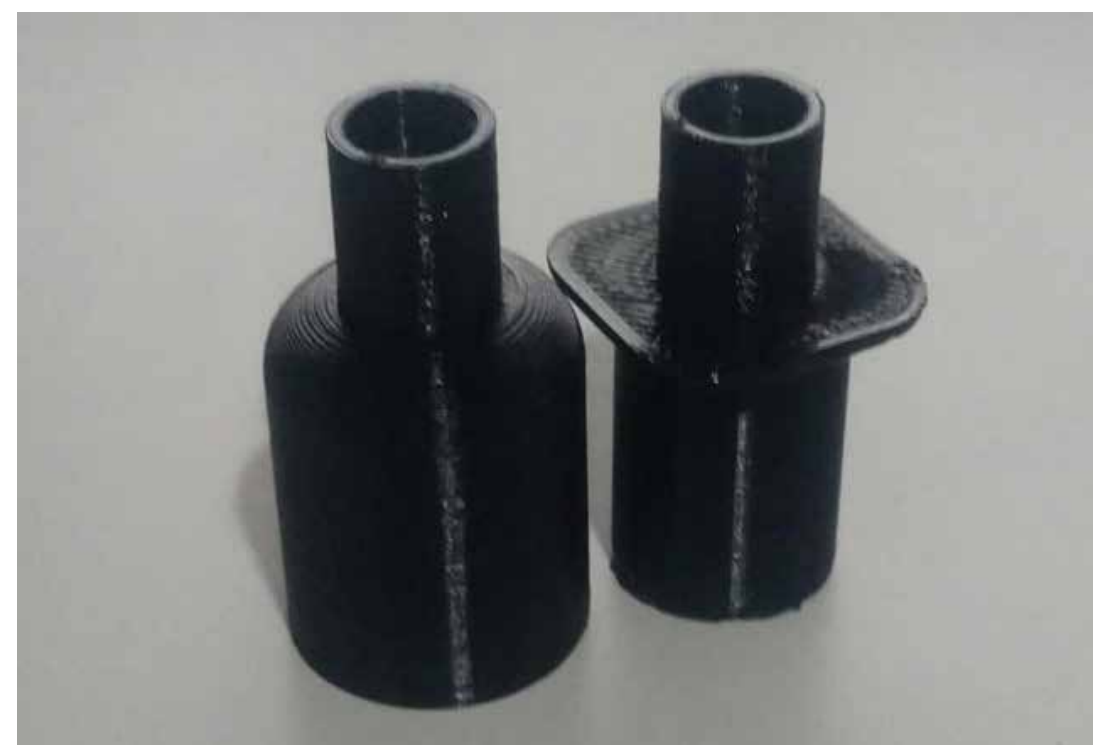

impressão em formato 3D. No dia 22 de abril de 2020 foi apresentado o desenho preliminar dos dois conectores que compoem o dispositivo (Figuras 2 e 3 ) e já no dia 23 de abril de 2020 as pecas foram impressas em formato 3D (Figura 4).

No dia 24 de abril de 2020 foi realizado o teste dos conectores para checar a adaptabilidade e constatada a perfeita adaptação do conector 2 , no entando, sendo necessária a revisão do diâmetro interno do conector 1. Após o ajuste, este foi testado novamente no dia $26 \mathrm{de}$ abril de 2020, demonstrando perfeita adaptação à cânula de traqueostomia e, então, o dispositivo CONECTONY foi montado (Figura 5).

A fase posterior, em desenvolvimento, compreende testes adicionais, descrição da funcionalidade e definição de protocolos com instruções de instalação e utilização atendendo às determinações e recomendações dos órgãos e sociedades profissionais de assistência à saúde. A Figura 6 demonstra a aplicabilidade em uma cânula de traqueostomia e, ainda, a aplicação em uma máscara laríngea, outra possibilidade observada durante os testes de aplicabilidade.

\section{CONCLUSÃO}

Este projeto contou com a colaboração técnica do Grupo de Trabalho (GT) Multidisciplinar para Enfrentamento da COVID-19, instituido em fevereiro de 2020 pela Reitoria da UFRJ, permitindo concretizar a transformação do projeto conceito em um projeto implementável na prática clínica e no cuidado de Enfermagem.

A realização do projeto tendo como base os fundamentos do Disign Thinking e, ainda, contando com suporte de Tecnologias de Informação e Comunicação para operacionalizar o trabalho remoto, consistiu em uma experiência singular, extremamente produtiva e gratificante, pois a integração interdisciplinar de ideias, saberes e conhecimentos permitiu a produção da inovação tecnológica em curtíssimo período (apenas cinco dias), 


\section{Figura 5. Dispositivo CONECTONY. Rio de Janeiro, RJ, Brasil, 2020.}

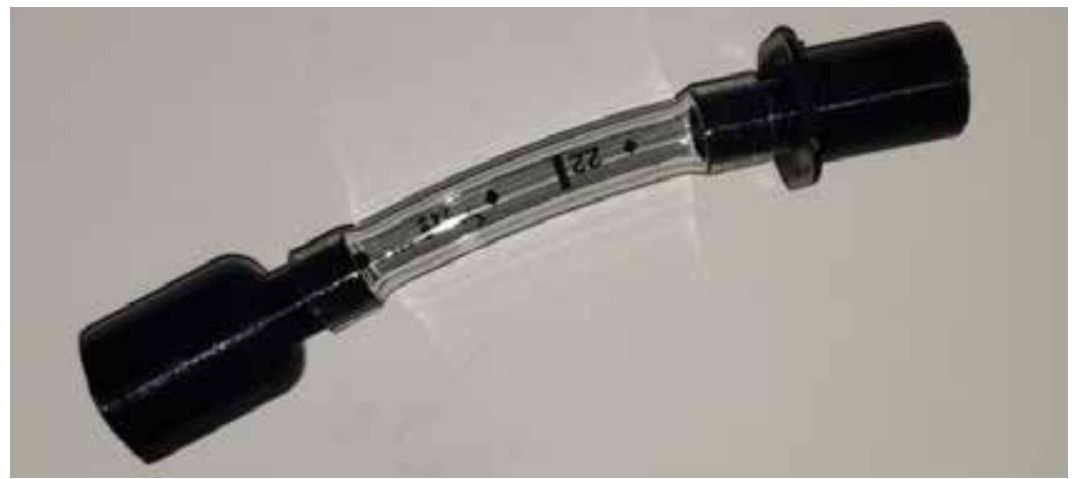

Figura 6. Aplicabilidade do dispositivo CONECTONY. Rio de Janeiro, RJ, Brasil, 2020.
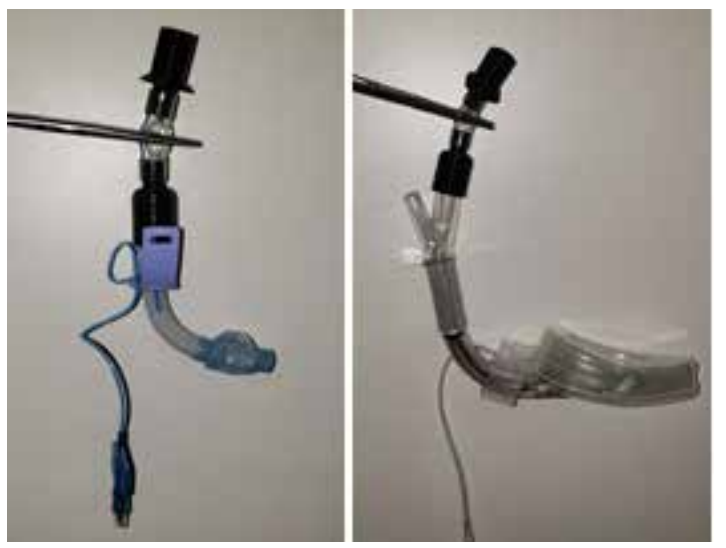

em tempo hábil para atender a demanda de utilização no contexto da pandemia de COVID-19.

O protótipo final, impresso em 3D, demonstrou perfeita adaptabilidade à cânula de traqueostomia e aos demais conectores e circuitos de assistência ventilatória, constituindo-se em importante dispositivo na estratégia de minimizar riscos de exposição profissional, permitindo atender às recomendações técnicas de clampeamento dos dispositivos de via aérea avançada no contexto da pandemia por COVID-19.

Incorporar a utilização do dispositivo CONECTONY à prática assistencial e ao cuidado de Enfermagem aos pacientes traqueostomizados tem potencial para conferir maior segurança aos profissionais de saúde, minimizando o risco de contaminação por aerossolização de microrganismos no ambiente, constituindo a indicação de seu uso, de extrema relevância, ainda maior neste momento de pandemia por COVID-19.

Possíveis ajustes podem ser necessários, tanto relacionados ao design do dispositivo, quanto à necessidade de revisão de processos de utilização, o que requerer a continuidade do estudo, monitoração e aperfeiçoamento. -

\section{REFERÊNCIAS}

1. Giwa A, Desai A.Novel coronavirus COVID-19: an overview for emergency clinicians. Emerg Med Pract [Internet]. 2020 Feb [acesso em 20 mai 2020]; 27;22(2Suppl2):1-21. Disponivel em: https://www.unboundmedicine.com/medline/citation/32105049/Novel_coronavirus_COVID_19:_an_overview_for_emergency_clinicians.

2. Associação Brasileira de Medicina de Emergência, Associação de Medicina Intensiva Brasileira (BR). Recomendações para Intubação Orotraqueal em pacientes portadores de COVID-19 Versão n. ${ }^{0}$ 3/2020 [Internet]. Atualizada de 10 de abril de 2020. Associação Brasileira de Medicina de Emergência (ABRAMEDE) e Associação de Medicina Intensiva Brasileira (AMIB) [acesso em 20 mai 2020]. Disponivel em: http://abramede.com.br/wp-content/uploads/2020/04/Recomendacoes-IOT-FINAL-REVISAO-100420.pdf.

3. American Heart Association. Coronavirus (COVID-19) Resources for CPR Training [Internet]. 2020 [acesso em 20 mai 2020]. Disponivel em: https://cpr.heart.org/en/resources/coronavirus-covid19-resourcesfor-cpr-training.
4. Cook TM, El Boghdadly K, McGuire B, McNarry AF, Patel A, Higgs $A$. Consensus guidelines for managing the airway in patients with COVID 19. Anaesthesia [Internet]. 2020. Disponível em: https://onlinelibrary.wiley.com/doi/pdfdirect/10.1111/ anae. 15054.

5. Tanabe LP, Kobayashi RM. Profile, competencies, and digital fluency of nurses in the Professional Improvement Program. Rev Esc Enferm USP [Internet]. 2013 [cited 2014 Aug 23]; 47(4):943-9. Available from: http://www.scielo.br/pdf/reeusp/ v47n4/en_0080-6234- reeusp-47-4-0943.pdf.

6. Cruz T. Sistemas de informações gerenciais: tecnologias da informação e a empresa do século XXI. 4. ed. São Paulo: Atlas; 2014.

7. Haubert B, Schreiber D, Pinheiro CMP. Combinando o design thinking e a criatividade no processo de inovação aberta. Revista Gestão e Planejamento [Internet]. 2019 jan./dez. [acesso em 20 mai 2020]; 20:73-89. Disponivel em: https://www.researchgate. net/publication/332903097. 